\title{
A Neural Network Assisted Cascade Control System for Air Handling Unit
}

\author{
Chengyi Guo, Qing Song, Member, IEEE, and Wenjian Cai, Member, IEEE
}

\begin{abstract}
In the centralized heating, ventilating and air-conditioning (HVAC) system, air handling units (AHUs) are traditionally controlled by single-loop proportional-integral-derivative (PID) controllers. The control structure is simple, but the performance is usually not satisfactory. In this paper, we propose a cascade control strategy for temperature control of AHU. Instead of a fixed PID controller in the classical cascade control scheme, a neural network (NN) controller is used in the outer control loop. This approach not only overcomes the tedious tuning procedure for the inner and outer loop PID parameters of a classical cascade control system, but also makes the whole control system be adaptive and robust. The multilayer $\mathrm{NN}$ is trained online by a special training algorithm-simultaneous perturbation stochastic approximation (SPSA)-based training algorithm. With the SPSA-based training algorithm, the weight convergence of the NN and stability of the control system is guaranteed. The novel cascade control system has been implemented on an experimental HVAC system. Testing results demonstrate the effectiveness of the proposed algorithm over the classical cascade control system.
\end{abstract}

Index Terms-Air handling units, cascade control, neural networks (NNs), simultaneous perturbation stochastic approximation (SPSA).

\section{INTRODUCTION}

$\mathbf{I}$ $\mathrm{N}$ heating, ventilating, and air-conditioning (HVAC) systems, the function of air handling units (AHU) is to transfer cooling load from air loop to chilled water loop by forcing airflow over the cooling coil and into the space to be conditioned. The performance of AHU directly influences the performance of HVAC systems. Traditionally, AHUs are controlled by singleloop proportional-integral-derivative (PID) type controllers, as shown in Fig. 1, due to the relatively simple structure [1]. However, in cases where requirements of the environment for equipments are very high, such as clean rooms (typically, temperature and humidity specifications require error tolerances of $\pm 0.5^{\circ}$ $\mathrm{C}$ and $2 \% \mathrm{RH}$, respectively), it would be desirable to integrate PID controllers into complex control structures to achieve better performance.

In this paper, we propose a cascade control strategy for AHU temperature control system. Cascade control algorithms are constructed by two control loops: an inner loop with fast dynamic to eliminate input disturbances and an outer loop to

Manuscript received March 16, 2005; revised October 11, 2005. Abstract published on the Internet November 30, 2006.

The authors are with the School of Electrical and Electronic Engineering, Nanyang Technological University, Singapore 639798 (e-mail: eqsong@ntu. edu.sg).

Color versions of one or more of the figures in this paper are available online at http://ieeexplore.ieee.org.

Digital Object Identifier 10.1109/TIE.2006.888809

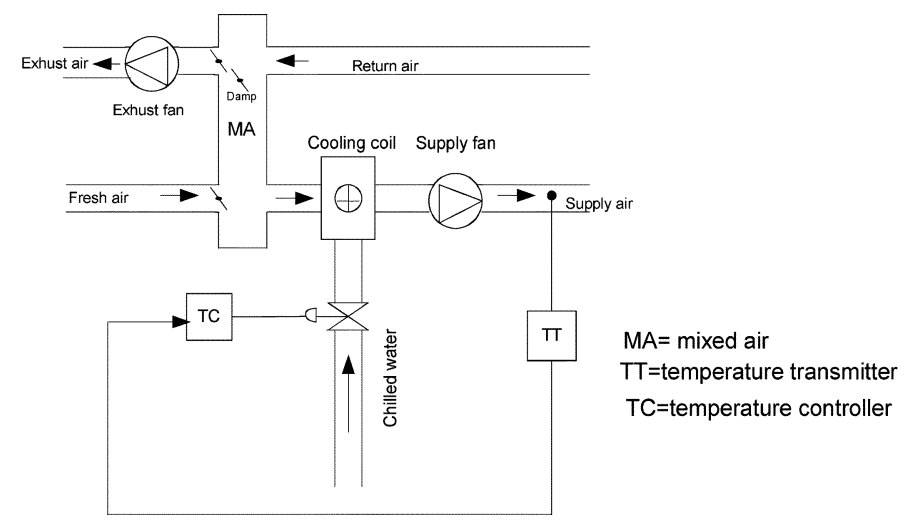

Fig. 1. Schematic diagram of conventional AHU control system (single control loop).

regulate output performance [2], [3]. It is particularly useful when there are significant dynamic differences between the control and process variables, tight control actions can be achieved by using an intermediate signal that responds faster than the original control signal. The cascade temperature control strategy of AHU can be implemented by selecting the flow rate of chilled water as an intermediate control signal that has fast response to the valve position change. Traditionally, the cascade control scheme is constructed as: a P or PI controller for the inner loop; a PI or PID controller for the outer loop. However, in most practical cases, such a cascade control system cannot deal adequately with the robustness issues [4]. In particular, when the inner loop part of the process is subject to structural and/or parametric variations, the control system does not guarantee an acceptable performance. It is desirable to enhance the performance robustness of the classical cascade control system and make it adaptive to both the plan nonlinearity and dynamic change. Furthermore, tuning the PID controllers requires effective process identification and controller design rules. For cascade control, it is difficult to obtain the well-tuned PID parameters for both the outer loop and the inner loop simultaneously. If retuning of the system dynamic is needed because of the environment change or aging effect, the situation may be even worse.

To enhance the robustness performance of AHU and surmount the problems for cascade control system mentioned above, in this paper, a neural network (NN)-assisted cascade control system is proposed. Instead of a fixed PI or PID controller, a NN controller is used in the outer control loop.

NNs have been increasingly applied to the control of a nonlinear system and are now finding application in a HVAC system. Miller and Seem [5] investigated a NN approach to 
the prediction of return time from night setback. The network in an open-loop control mode was used to predict the startup time from measured values of the outside temperature and the initial room temperature. Curtiss et al. [6] applied an adaptive, $\mathrm{NN}$-based control scheme to a hot water heating coil. The NN, which is used in the feedback loop, models the dynamic behavior of the coil and predicts future process outputs. An RBF neural network controller was designed to control the heating coil in [7]. The neural control scheme is capable of compensating for plant nonlinearity and adapting online to degradation in the plant. However, $\mathrm{NN}$ training algorithms are sensitive to disturbances and may not be able to obtain an optimal performance without guaranteed stability [8]. In the NN-assisted cascade control system studied in this paper, we use the simultaneous perturbation stochastic approximation-based training algorithm proposed in [9]. With the SPSA-based training algorithm, the weight convergence of the NN and stability of the control system is guaranteed. We apply the new cascade control scheme to the air temperature control of the AHU, dealing with the chilled water flow rate fluctuation, which is the main disturbance in a typical chilled water distribution system. Testing results obtained from both single-loop and cascade control strategies are presented to demonstrate the performance of the new control scheme. It shows that, with the proposed cascade control scheme, the temperature control performance of AHU is significantly improved for both temperature set-point tracking and chilled water flow rate disturbances rejection.

\section{Description OF AHU CONTROL System}

The cooling only AHU system is shown in Fig. 1, it consists of cooling coil, air dampers, fans, chilled water pumps, and valves. The fresh air, depending on the outdoor air damper settings, may be mixed with the air passing through the recirculation air damper. Return air is drawn from the zones by the return fan and is either exhausted or recirculated, depending on the position of the mixing box dampers. The temperature and flow rate of the outdoor and recirculation air streams determine the conditions at the exit of the mixed air plenum. Air exiting the mixed air plenum pass through the cooling coils. After being conditioned in the coils, the air is distributed to the zones through the supply air ductwork. The supply air temperature is measured through the downstream airflow of the supply fan. For the off-coil temperature control loop, the manipulated variable is the valve opening position that controls the chilled water supplied to the cooling coil. The measured process output is the off-coil downstream temperature of the supply fan. The objective of the AHU is to maintain the supply air temperature at the set-point value.

Let $G_{p}$ and $G_{c}$ denote the dynamic models of the AHU process and the fixed PID temperature controller in the single control loop, respectively, the block diagram of the conventional temperature control system is shown in Fig. 2. The drawback of the conventional PID controller $G_{C}$ is that it has a single degree-of-freedom in tuning. Considering a central HVAC plant, as shown in Fig. 3, where the main chilled water distribution pipe may supply chilled water to many AHUs. Therefore, flow rate change in one AHU branch will affect all other loops.

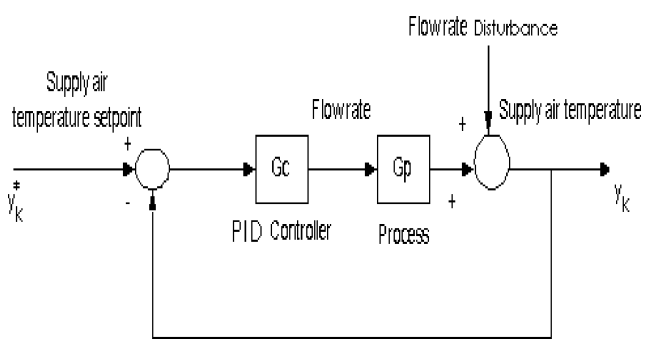

Fig. 2. Block diagram of AHU's supply air temperature AHU control system (single control loop).

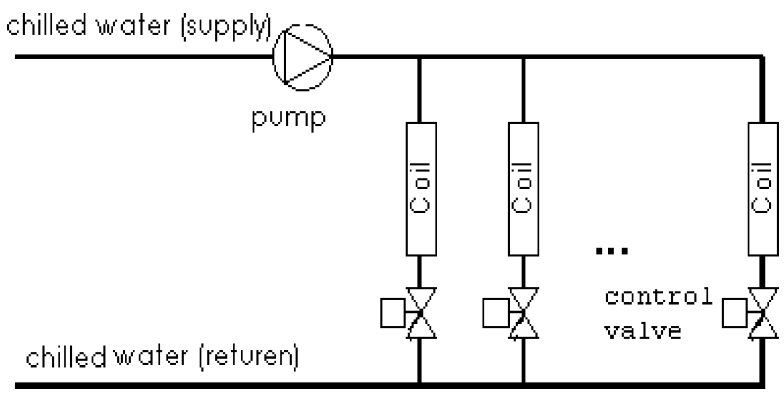

Fig. 3. Typical chilled water distribution system.

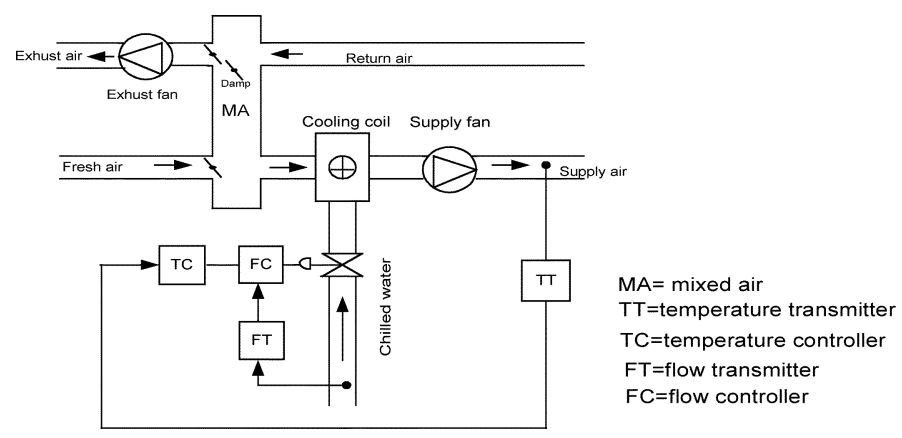

Fig. 4. Schematic diagram of cascade control for supply air temperature.

When a conventional single-loop temperature control scheme is used with the output of the temperature controller applied directly to the control valve, no correction will be made until its effect reaches the temperature measuring elements. Thus, there is a considerable lag in correcting flow rate disturbances.

To improve the response of the simple feedback control, with a cascade control technique using a separate flow rate control loop, the flow rate controller will correct any change in the chilled water flow rate. Fig. 4 shows a schematic diagram of the cascade control system for supply air temperature, while the control block diagram is shown in Fig. 5, where $G_{c 1}, G_{c 2}, G_{p 1}$, and $G_{p 2}$ stand for the temperature controller, chilled water flow rate controller, AHU process, and valve process, respectively.

\section{NN-Assisted CASCADE CONTROL SCHEME AND THE SPSA-BASED TRAINING ALGORITHM}

To deal with the system nonlinearity and uncertainty (especially the dynamic change of the inner loop), instead of the traditional PI or PID controller, we employ a NN controller for the outer loop of AHU temperature control. As shown in Fig. 6, the equivalent outer loop process $G_{P 0}$ consists of the inner loop 


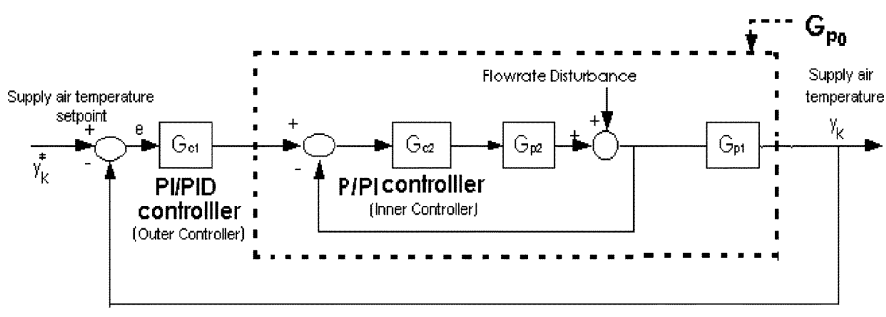

Fig. 5. Block diagram of conventional cascade control.

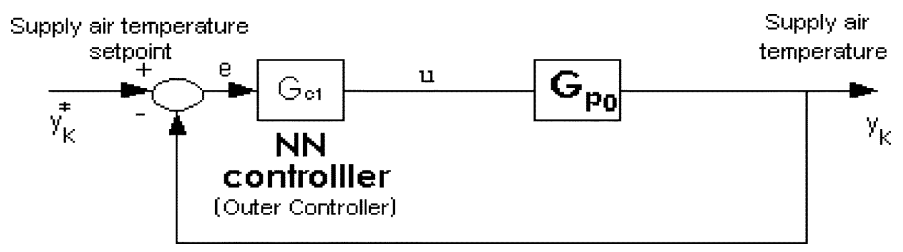

Fig. 6. Block diagram of the outer control loop.

P/PI controller $G_{c 2}$, the inner loop valve process $G_{p 2}$, and the AHU process $G_{p 1}$ (refer to Fig. 5).

\section{A. NN-Assisted Cascade Control Scheme}

Fig. 7 shows the structure of the $\mathrm{NN}$-assisted cascade control system. It can be seen that, the outer control loop comprises a fixed proportional feedback controller and a NN controller that is trained to approximate the inverse dynamics of the plant. With $k$ as the time index, the dynamic of the outer control loop can be represented as a single-input-single-output form as the following:

$$
\begin{array}{r}
y_{k}=f\left(y_{k-1}, y_{k-2}, \ldots y_{k-m_{y}}, u_{k-d-1}, u_{k-d-2}\right. \\
\left.\ldots u_{k-d-m_{u}}\right)+u_{k-d}+\varepsilon_{k}
\end{array}
$$

where $y$ is the output, $u$ is the control input, and $d$ is the relative degree of the system. Particularly, for the outer loop of AHU temperature control studied in this paper, $y$ is the supply air temperature, and $u$ is the set-point value of chilled water flow rate in the inner control loop. $f($.$) is the unknown dynamic nonlinear$ function, and $\varepsilon_{k}$ denotes a bounded overall noise signal of the control system. $m_{y}, m_{u}$ are integers and $m_{y} \geq m_{u}$.

The set-point tracking error is

$$
s_{k}=y_{k}-y_{k}^{*}
$$

where $y_{k}^{*}$ is the command input. Define the control signal as

$$
u_{k-d}=-\hat{f}(.)+y_{k}^{*}+K_{P} s_{k-1}
$$

where $\hat{f}($.$) is estimate of the nonlinear function f($.$) by the NN$ to be defined later, and $K_{P}$ denotes the proportional gain of the fixed controller. We have the control signal at time step $k$ as

$$
\begin{aligned}
u_{k}= & -\hat{f}(.)+y_{k+d}^{*}+K_{P} s_{k+d-1} \\
= & -\hat{f}\left(y_{k+d-1}, y_{k+d-2}, \ldots y_{k+d-m_{y}}, u_{k-1}, u_{k-2}, \ldots u_{k-m_{u}}\right) \\
& +y_{k+d}^{*}+K_{P} s_{k+d-1} .
\end{aligned}
$$

Note that the equation above shows that the control system becomes noncausal when $d>1$. However, for the proposed controller design, this issue can be typically addressed by introducing a linear observer with the extra observation of the difference signals

$$
y_{k-1+d}=\dot{y}_{k+d-2} T+y_{k+d-3}
$$

where $T$ is the sampling time, and $\dot{y}$ is the differential signal of output $y$. In the cases when $\dot{y}$ is not measurable, it can be approximated by the output signal.

For example

$$
\dot{y}_{k} \approx \frac{\left(y_{k}-y_{k-1}\right)}{T} .
$$

The estimation error vector of the NN can be presented as

$$
e_{k}=f_{k-1}-\hat{f}_{k-1}+\varepsilon_{k} .
$$

The NN control structure is shown in Fig. 7 with $m_{y}=2$, $m_{u}=0$, and $d=2$. Note that, for the NN training, the estimation error $e_{k}$ may not be directly measurable, so we should use the tracking error to generate it based on the closed-loop relationship via (1)-(3) and (6)

$$
e_{k}=\left[1-z^{-1} K_{P}\right] s_{k} .
$$

The output of a three-layered NN can be presented as

$$
\hat{f}_{k}=H\left(w_{k}, x_{k}\right) v_{k}^{T}
$$

where the input vector $x_{k} \in R^{m}$ of the $\mathrm{NN}$ is

$$
x_{k}=\left[y_{k-1}, y_{k-2}, \ldots y_{k-m_{y}}, u_{k-d-1}, u_{k-d-2}, \ldots u_{k-d-m_{u}}\right]^{T}
$$

with $m=m_{y}+m_{u} \cdot v_{k} \in R^{n_{h}}$ is the weight vector of the output layer, $n_{h}$ is the numbers of neurons in the hidden layers of the network, $w_{k} \in R^{n_{h} \times m}$ is the adjustable weight matrix of the hidden layer, and $H\left(w_{k}, x_{k}\right)$ is the nonlinear activation function vector

$$
H\left(w_{k}, x_{k}\right)=\left[h\left(w_{k, 1}^{T} x_{k}\right) h\left(w_{k, 2}^{T} x_{k}\right) \ldots h\left(w_{k, n_{h}}^{T} x_{k}\right)\right]
$$

where $h($.$) is the nonlinear activation function$

$$
h(x)=\frac{1}{\left(1+e^{-4 \lambda x}\right)}
$$

where $\lambda>0$ is the so-called gain parameter of the threshold function.

\section{B. SPSA-Based Training for NN}

The SPSA algorithm proposed by Spall [10], [11] is a numerically efficient stochastic optimization algorithm. Unlike the conventional gradient-based optimization methods, which evaluate the gradient of the objective function by perturbing each decision variable separately (as in the standard two-sided finite difference approximation), the SPSA formalism approximates the 


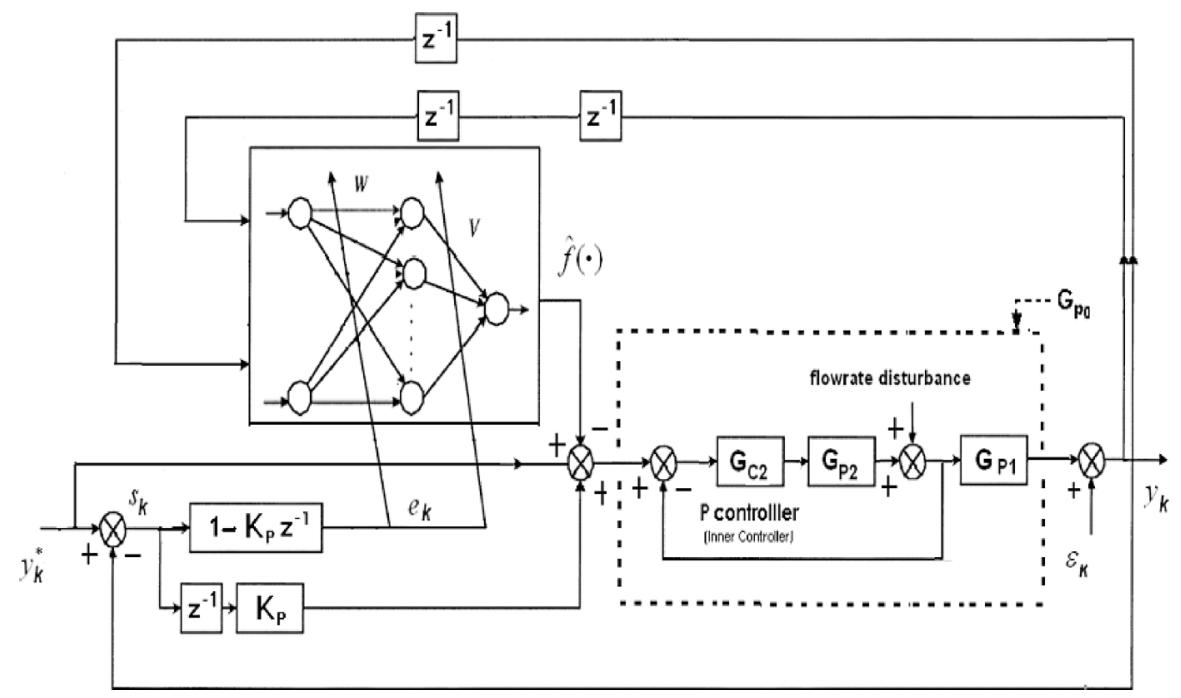

Fig. 7. Structure of the NN-assisted cascade control scheme (with $m_{y}=2, m_{u}=0, d=2$ ).

gradient by perturbing all the variables simultaneously. The optimization objective underlying the SPSA is defined as: finding the optimal decision variable vector $\theta^{*}$, such that it simultaneously minimizes the objective function $L(\theta)$. The SPSA implementation is iterative that begins with a randomly initialized solution vector. The basic SPSA formalism stipulates that the objective function to be minimized should be differentiable. For the minimum point $\theta^{*}$, the gradient of the objective function $L(\theta)$ attains zero magnitude. That is

$$
g\left(\theta^{*}\right)=\left.\frac{\partial L(\theta)}{\partial \theta}\right|_{\theta=\theta^{*}}=0 .
$$

The estimate of the decision variable vector $\theta$ is updated iteratively as

$$
\hat{\theta}_{k}=\hat{\theta}_{k-1}-a_{k} \hat{g}_{k}\left(\hat{\theta}_{k-1}\right)
$$

where $a_{k}$ is the learning rate and $\hat{g}_{k}\left(\hat{\theta}_{k-1}\right)$ is the approximation of the gradient function with

$$
\hat{g}_{k}\left(\hat{\theta}_{k-1}\right)=\frac{L\left(\hat{\theta}_{k-1}+c_{k} \Delta_{k}\right)-L\left(\hat{\theta}_{k-1}-c_{k} \Delta_{k}\right)+\varepsilon_{k}^{\theta}}{2 c_{k}} r_{k}
$$

and where $\varepsilon_{k}^{\theta}$ is the measurement disturbance, as defined in [10]. In the above equation, $\Delta_{k} \in R^{p}$ is a random directional vector For hidden layer weights update:

that is used to stimulate the weight vector simultaneously, and $c_{k}>0$ is a sequence of positive number satisfying certain regularity conditions [10], [11]. The random vector $\Delta_{k}$ is generated via Monte Carlo according to conditions specified in [10] or [11]. If the $i$ th element of $\Delta_{k}$ is denoted as $\Delta_{k i}$, then the sequence of $r_{k} \in R^{p}$ is defined as

$$
r_{k}=\left[\frac{1}{\Delta_{k_{1}}} \ldots \cdots \frac{1}{\Delta_{k_{p}}}\right] .
$$

Implementation of SPSA may be easier than other stochastic optimization methods (such as forms of the genetic algorithm), since there are fewer algorithm coefficients that need to be specified, and there are some published guidelines providing insight into how to pick the coefficients in practical applications [12]. When applying the SPSA to the three-layer NN training, we define the overall estimate decision vector as $\hat{\theta}_{k}=\left[\hat{\theta}_{k}^{v T} \hat{\theta}_{k}^{w T}\right]^{T} \in R^{p}$ with $p=n_{h}+n_{h} m$, where $\hat{\theta}_{k}^{v}=v_{k}$ and $\hat{\theta}_{k}^{w}=\left[\begin{array}{llll}w_{k, 1}^{T} & w_{k, 2}^{T} & \cdots & w_{k, n_{h}}^{T}\end{array}\right]$. Then, the output of a three-layer NN can be presented as

$$
\hat{f}_{k}=H\left(\hat{\theta}_{k}^{w}, x_{k}\right) \hat{\theta}_{k}^{v} .
$$

It should be noted that, for multilayer NNs, it might not be possible to update all the estimated parameters with a single gradient approximation function (13) to meet the stability requirement. Therefore, the estimate parameter vectors $\hat{\theta}_{k}^{v}$ and $\hat{\theta}_{k}^{w}$ are updated separately in the SPSA algorithm using different gradient approximation functions as in the standard backpropagation training algorithm. The SPSA-based training algorithm for the output and hidden layer of the multilayer $\mathrm{NN}$ can be further rewritten as follows:

For output layer weights update:

$$
\begin{aligned}
\hat{\theta}_{k}^{v} & =\hat{\theta}_{k}^{v}-a_{k} \hat{g}\left(\hat{\theta}_{k-1}^{w}, \hat{\theta}_{k-1}^{v}, \Delta_{k}^{v}\right) \\
& =\hat{\theta}_{k-1}^{v}+a_{k} \frac{s_{k}^{T}\left(1-K_{P} z^{-1}\right) H\left(\hat{\theta}_{k-1}^{w}, x_{k-1}\right) 2 \Delta_{k}^{v}}{\rho_{k}^{v}} r_{k}^{v} .
\end{aligned}
$$

$$
\begin{aligned}
\hat{\theta}_{k}^{w} & =\hat{\theta}_{k}^{w}-a_{k} \hat{g}\left(\hat{\theta}_{k-1}^{w}, \hat{\theta}_{k-1}^{v}, \Delta_{k}^{v}\right) \\
& =\hat{\theta}_{k-1}^{w}+a_{k} \frac{s_{k}^{T}\left(1-K_{P} z^{-1}\right)\left(f_{k-1}^{w+}-f_{k-1}^{w-}\right)}{c_{k} \rho_{k}^{w}} r_{k}^{w}
\end{aligned}
$$

where $\rho_{k}^{v}=\mu \rho_{k-1}^{v}+\max \left(4\left\|H\left(\hat{\theta}_{k-1}^{w}, x_{k-1}\right)\right\|^{2}\left\|\Delta_{k}^{v}\right\|^{2}\left\|r_{k}^{v}\right\|^{2}\right.$ $, \bar{\rho})$ with $\bar{\rho}>0, \mu \in(0,1)$

$$
\begin{aligned}
\rho_{k}^{w} & =\mu \rho_{k-1}^{w}+\max \left(\left\|\hat{f}_{k-1}^{w-}-\hat{f}_{k-1}^{w+}\right\|^{2}\left\|r_{k}^{w}\right\|^{2}, \bar{\rho}\right) \\
\hat{f}_{k-1}^{w+} & =H\left(\hat{\theta}_{k-1}^{w}+c_{k} \Delta_{k}^{w}, x_{k-1}\right) \hat{\theta}_{k-1}^{v} \\
\hat{f}_{k-1}^{w-} & =H\left(\hat{\theta}_{k-1}^{w}-c_{k} \Delta_{k}^{w}, x_{k-1}\right) \hat{\theta}_{k-1}^{v} .
\end{aligned}
$$

Remark: The derivation of the two weight update rules (16)-(17) is given in [9]. The reader should refer to this refer- 


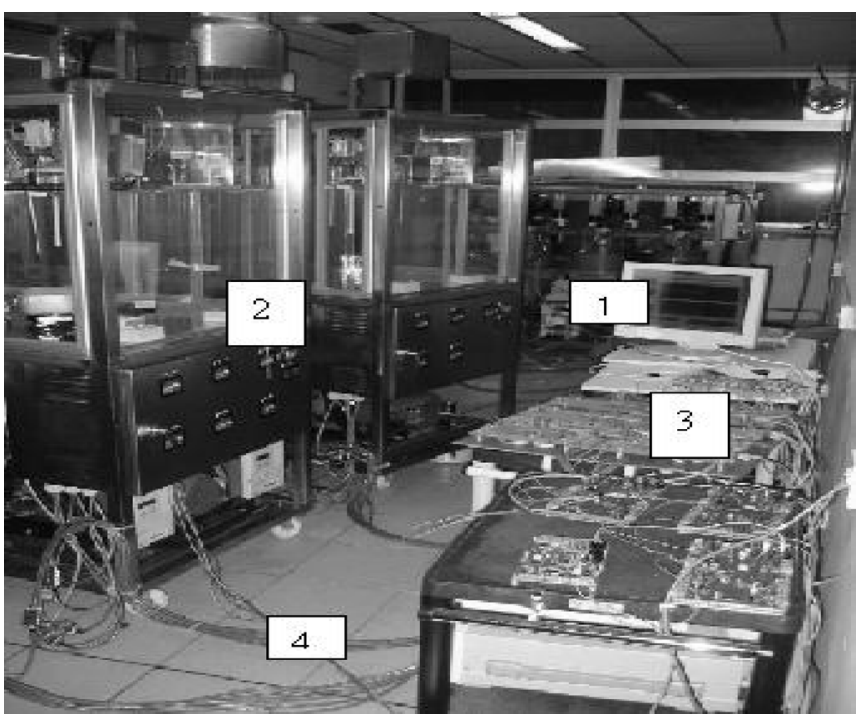

Fig. 8. Measurement and control apparatus of HVAC pilot system.

ence for more details. Theorems 1 and 2 and their proof in [9] show that the introduction of the normalization factor, $\rho_{k}^{v}$ and $\rho_{k}^{w}$, guarantees input-output stability of the closed outer control loop, as well as the convergence of the neural weights based on the conic sector theory, a smaller learning rate and larger number of neurons may increase the stability of the control system. It is also shown in [9] that the parameter $c_{k}$ does not affect the system's stability.

The SPSA-based online training algorithm can be summarized as follows (refer to Fig. 7).

Step 1) Form the new input vector $x_{k-1}$ of the NN defined in (8).

Step 2) Calculate the NN output $\hat{f}_{k-1}$ by using the input state $x_{k-1}$ and the existing or initial weights of the network in the first iteration.

Step 3) The control input $u_{k-1}$ is calculated based on (3).

Step 4) The new measurement of the system dynamics is taken and the measurable tracking error signal $s_{k}$ is fed through a fixed filter to produce the implicit training error signal $e_{k}$ of the network according to (7).

Step 5) The tracking error $s_{k}$ is used directly to train the $\mathrm{NN}$ and calculates the new weights $\hat{\theta}_{k}^{v}$ and $\hat{\theta}_{k}^{w}$ by using learning law in (16) and (17) for the output and hidden layers, respectively, of the next iteration.

Step 6) Go back to Step 1) to continue the iteration.

\section{REAL-TIME EXPERIMENTS AND RESULTS}

\section{A. Experiment Setup}

The test is conducted on a pilot centralized HVAC system, as shown in Fig. 8, where 1, 2, 3, and 4 indicate the components of computer controller, HVAC pilot plant, signal process board, and signal transmission cable, respectively. The system has three chillers, three zones with three AHUs, three cooling towers, and flexible partitions with up to 12 rooms. All motors (fans, pumps, and compressors) are controller by VSDs. The system is made very flexible to configure these three units to

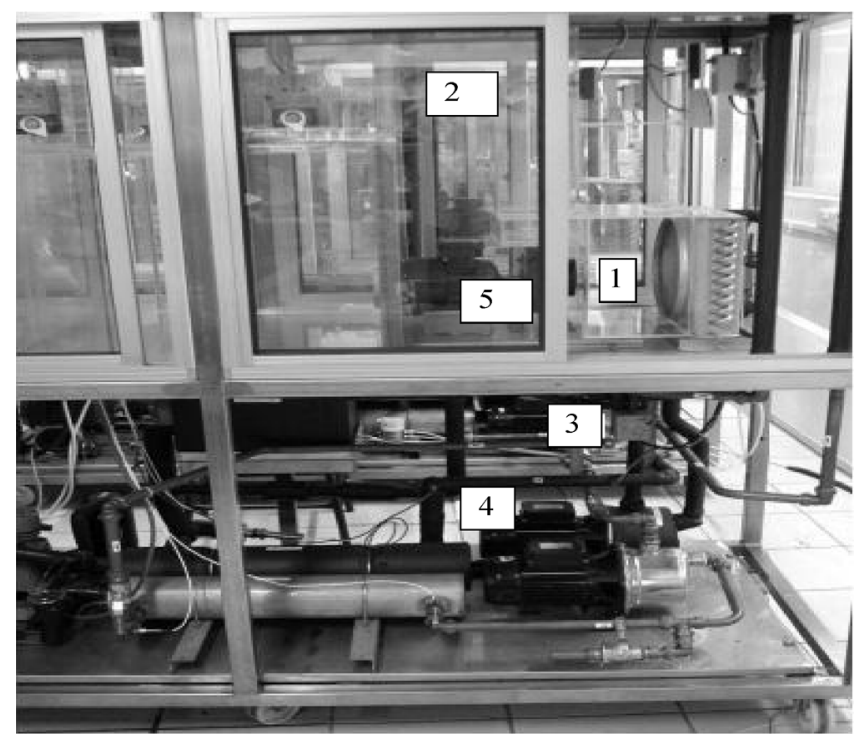

Fig. 9. AHU control system.

form different HVAC schemes. The cooling coils for the system are two rows with the dimension of $25 \mathrm{~cm} \times 25 \mathrm{~cm} \times 8 \mathrm{~cm}$, as shown in Fig. 9. Here, 1, 2, 3, 4, and 5 indicate the location of cooling coil, off-coil temperature sensor, chilled water control valve, chilled water pump, and supply air fan, respectively.

\section{B. Experiment 1}

In the experiment, both off-coil temperature and chilled water flow rate are measured. The actuator is the chilled water control valve controlled by step motor. The chilled water temperature is controlled at around $8^{\circ} \mathrm{C}$ and supply air pressure is controlled to be constant by keeping the supply fan running at a constant speed. In order to compare the performances of single-loop control with cascade control, the overall process model of the AHU system is identified as $G_{p}(s)=[0.2 /(140 s+1)] e^{-49 s}$ and a single-loop PID controller based on Chien-Hrones-Reswick (CHR) tuning rule [13] is then designed and implemented with minor adjustments to obtain the best response. The PID parameters achieved are $K_{p}=17.5, K_{i}=0.18$, and $K_{d}=320$. For the cascade control strategy, the classical structure is designed first with fixed PI controller for inner loop and fixed PID controller for outer loop. The existing technique for tuning the classical cascade controllers is in two steps: first, the inner loop controller is tuned, subsequently, the inner loop controller is commissioned and the outer loop controller is tuned to complete the tuning process. Following the CHR tuning rule, with a further trial-and-error method, the optimal parameters for the real-time experiments are obtained. The PI controller of the inner loop: $K_{p 2}=22.21, K_{i 2}=0.88$; The PID controller of the outer loop: $K_{p 1}=0.8268, K_{i 1}=0.0044$, and $K_{d 1}=0.598$, respectively. For a fair comparison, the same PI controller is used in the inner loop for the $\mathrm{NN}$-assisted cascade control scheme. A three-layer NN is used in the outer control loop with 12 hidden layer neurons, all the initial weight values are chosen to be zero, the gain parameter in (4) is given as $K_{P}=0.8$. For the 


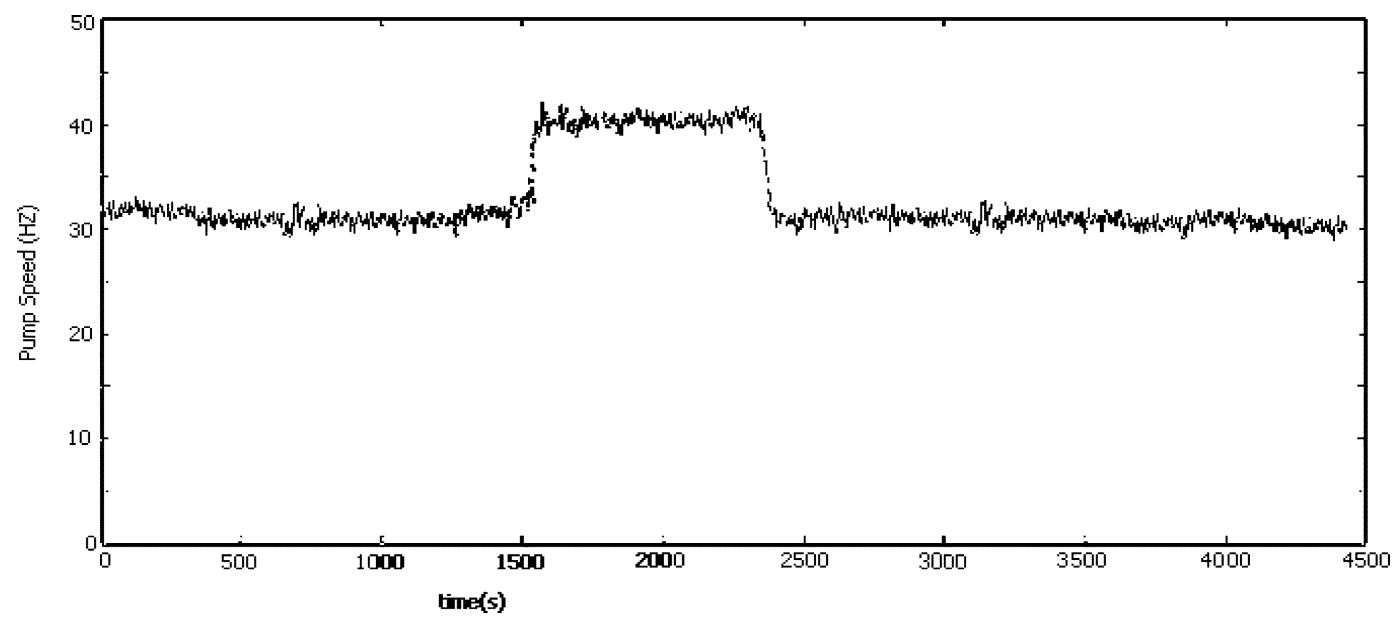

Fig. 10. Speed of chilled water pump.

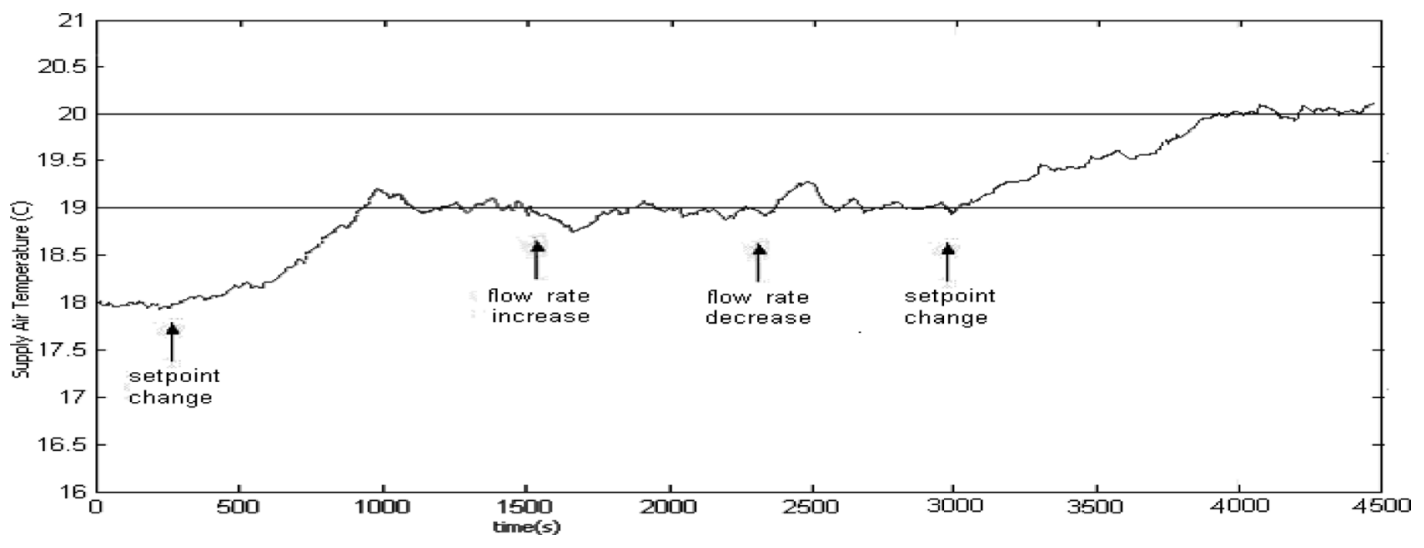

Fig. 11. Supply air temperature control performance of AHU in Experiment 1 (single loop PID control).

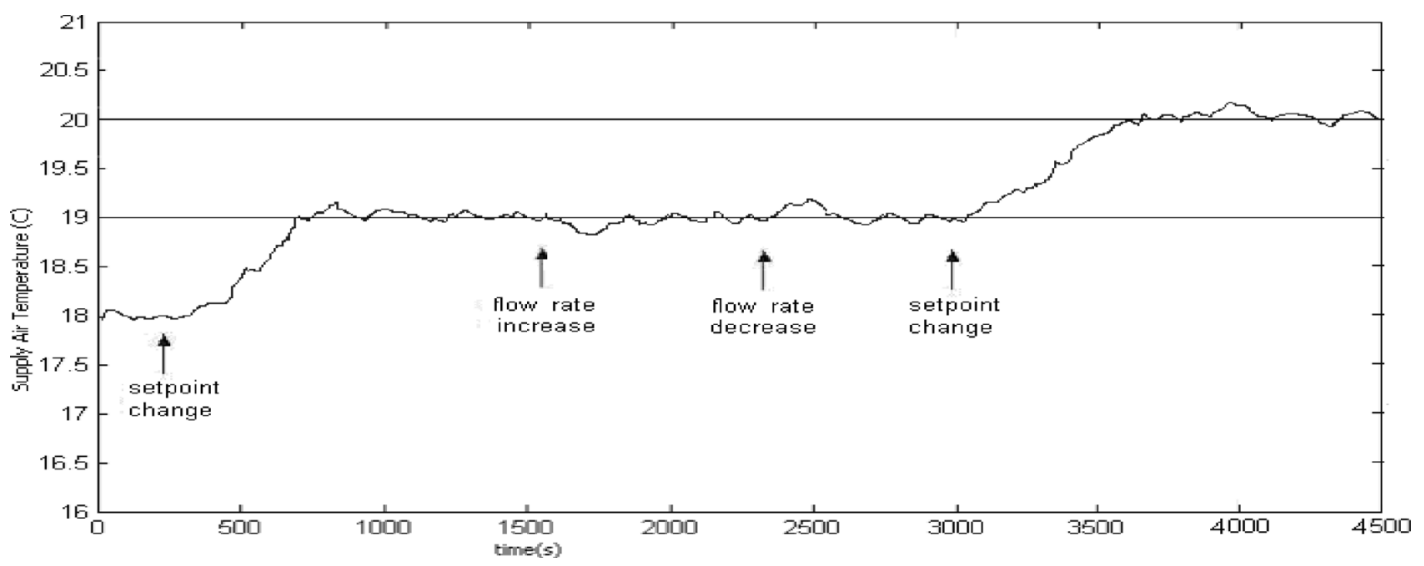

Fig. 12. Supply air temperature control performance of AHU in Experiment 1 (cascade control with PI inner loop controller and PID outer loop controller).

SPSA training algorithm, following the guideline given in [12], let $c_{k}=c / k^{r}$, here we choose $c=5$ and $r=0.101$ in the experiment.

Figs. 10-13 show the different response of single-loop control and cascade control for the case of temperature set-point change and chilled water flow rate fluctuation. The test is carried out as follows.

1) Stabilize the off-coil temperature at $18^{\circ} \mathrm{C}$.
2) Reset the off-coil temperature to $19^{\circ} \mathrm{C}$, while keeping the chilled water flow rate stable at time $t=250 \mathrm{~s}$.

3) For the same temperature set point, increase chilled water flow rate by increasing the chilled water pump speed at $t=1500 \mathrm{~s}$.

4) Keep set point unchanged and decrease the chilled water flow rate by decreasing the chilled water pump speed at $t=2300 \mathrm{~s}$. 


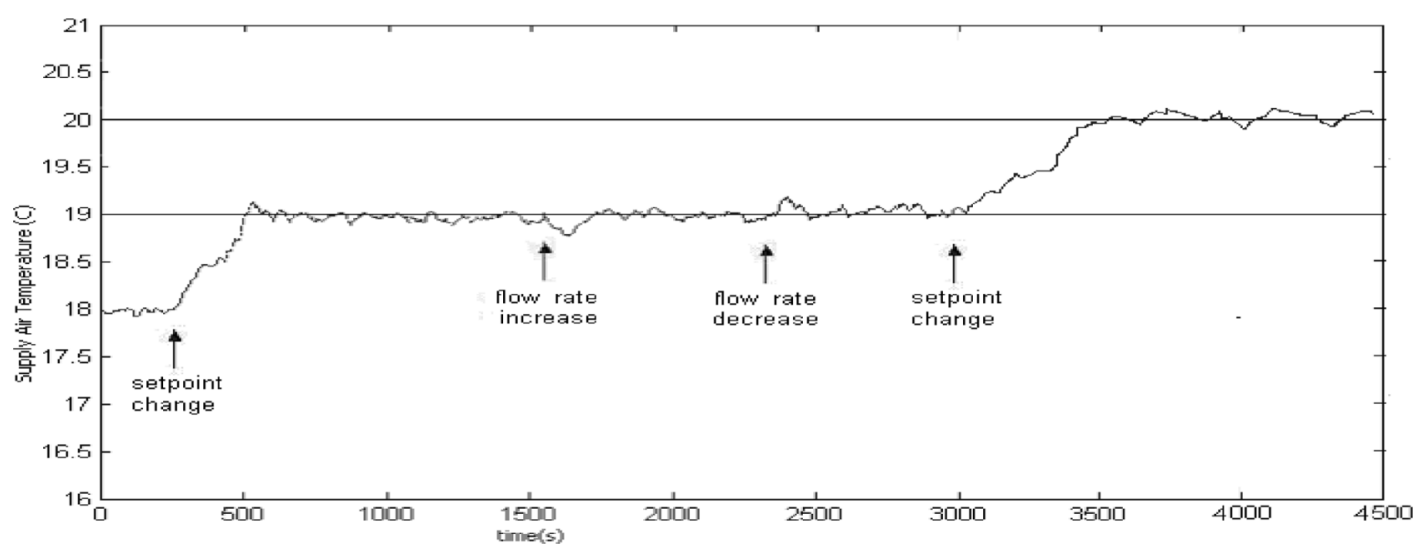

Fig. 13. Supply air temperature control performance of AHU in Experiment 1 (cascade control with PI inner loop controller and NN outer loop controller).

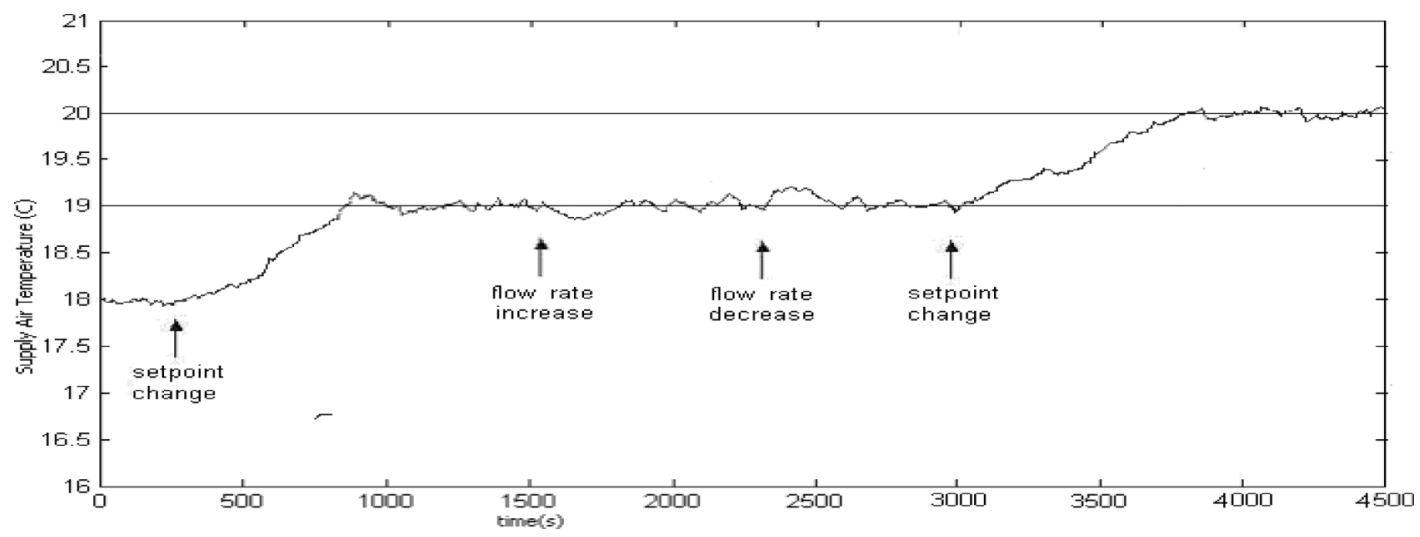

Fig. 14. Supply air temperature control performance of AHU in Experiment 2 (cascade control with PI inner loop controller and PID outer loop controller).

TABLE I

PERFORMANCE COMPARISON IN EXPERIMENT 1

\begin{tabular}{|c|c|c|c|c|}
\hline TESTING & CRITERIA & $\begin{array}{l}\text { SINGLE } \\
\text { LOOP (PID) }\end{array}$ & $\begin{array}{l}\text { CASCADE } \\
\text { (PI plus PID) }\end{array}$ & $\begin{array}{l}\text { CASCADE } \\
\text { (PI plus NNDC) }\end{array}$ \\
\hline $\begin{array}{l}\text { set-point change } \\
18^{\circ} \mathrm{C}-19^{*} \mathrm{C}\end{array}$ & Settlingtime $(s)$ & 670 & 450 & 280 \\
\hline $\begin{array}{l}\text { disturbance } \\
\text { (flowrate increase) }\end{array}$ & Settingtime (s) & 380 & 280 & 230 \\
\hline $\begin{array}{l}\text { disturbance } \\
\text { (flowrate decrease) }\end{array}$ & Suttingtime (s) & 350 & 240 & 190 \\
\hline $\begin{array}{c}\text { set-point change } \\
19 * \mathrm{C}-20^{\circ} \mathrm{C}\end{array}$ & Settingtime (s) & 800 & 580 & 400 \\
\hline
\end{tabular}

5) Reset the off-coil temperature to $20^{\circ} \mathrm{C}$, while keeping the chilled water flow rate stable at time $t=3000 \mathrm{~s}$.

The results are also summarized in Table I. It shows clearly that the proposed $\mathrm{NN}$-assisted cascade control system improves temperature control performance of AHU in terms of the setpoint tracking and disturbance rejection ability. Compared with the classical cascade control structure, by introducing the NN to compensate the systems nonlinearity, the proposed control system maintains a better performance at different operating points.
TABLE II

PERFORMANCE COMPARISON IN EXPERIMENT 2

\begin{tabular}{|c|c|c|c|}
\hline TESTING & CRITERIA & $\begin{array}{c}\text { CASCADE } \\
\text { (PI plus PID) }\end{array}$ & $\begin{array}{c}\text { CASCADE } \\
\text { (PI pluSNNC) }\end{array}$ \\
\hline $\begin{array}{c}\text { set-point change } \\
18^{\circ} \mathrm{C}-19^{\circ} \mathrm{C}\end{array}$ & Settlingtime (s) & 590 & 320 \\
\hline $\begin{array}{c}\text { disturbance } \\
\text { (flowrate increase) }\end{array}$ & Settlingtime (s) & 300 & 260 \\
\hline $\begin{array}{c}\text { disturbance } \\
\text { (flowrate decrease) }\end{array}$ & Settlingtime (s) & 270 & 200 \\
\hline $\begin{array}{c}\text { set-point change } \\
19^{\circ} \mathrm{C}-20^{\circ} \mathrm{C}\end{array}$ & Settlingtime (s) & 720 & 450 \\
\hline
\end{tabular}

\section{Experiment 2}

For the HVAC control system, in practice, an originally welltuned PID controller, after long-term running, because of the aging effect and system components degradation, the control performance of the controller will be greatly affected. In the second experiment, we continue to compare the performance between the classical cascade control and the NN-assisted cascade control scheme. The integral gain of the well-tuned PI controller in the inner control loop is decreased to 0.4 (i.e., $K_{i 2}=0.4$ ) to simulate the control performance degradation due 


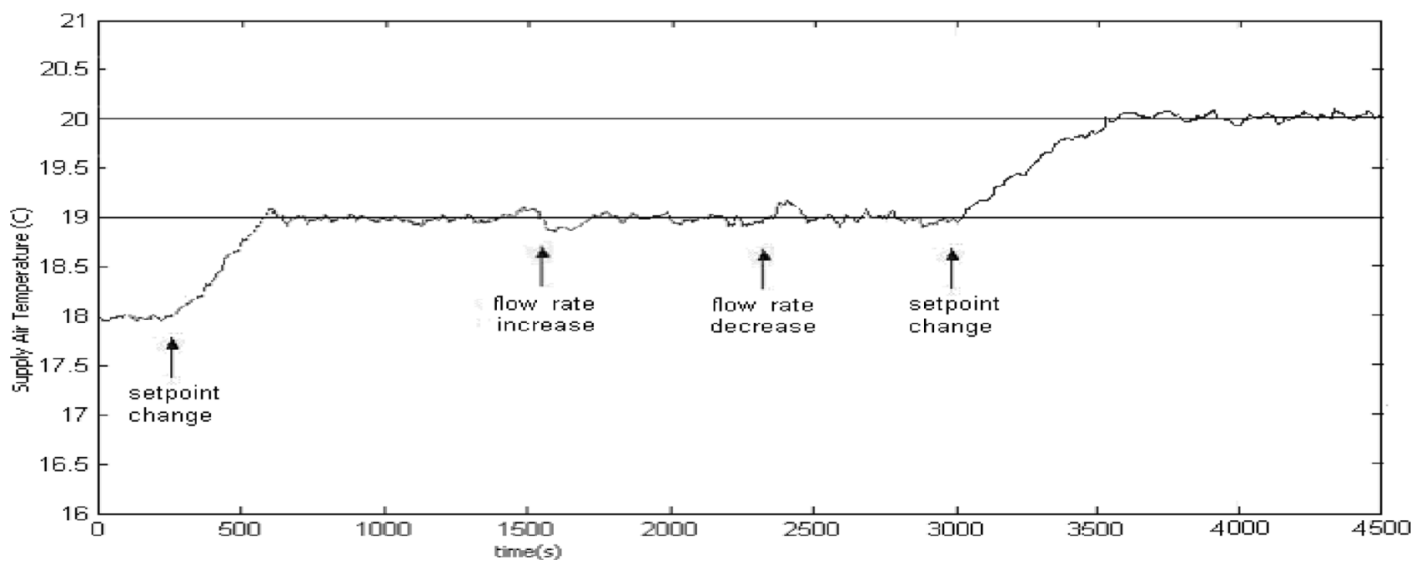

Fig. 15. Supply air temperature control performance of AHU in Experiment 2 (cascade control with PI inner loop controller and NN outer loop controller).

to the aging effect of long working hours, while other parameters of these two cascade control systems and test steps remain the same with the first experiment.

The experiment results are shown in the Figs. 14 and 15, and summarized in Table II. Comparing them with the results in the first experiment, for the $\mathrm{NN}$-assisted cascade control system, the control performance change is little in terms of the setpoint tracking and disturbance rejection ability, while the performance of the classical cascade controller is degraded a lot because of the inner loop PI controller's parameter change, which means, by integrating with the NN trained by the SPSA-based algorithm, the inner loop PI controller's performance degradation for aging effect can be compensated to a certain degree. This experiment also shows that, even the inner loop fixed controller is not tuned to its optimal value, the $\mathrm{NN}$-assisted cascade control system can still give a satisfactory performance because of its adaptiveness.

\section{CONCLUSION}

In this paper, the cascade control strategy for temperature control of AHU is introduced to deal with the chilled water flow rate fluctuation, which is the main disturbance in a typical chilled water distribution system and causes the deterioration of the temperature control performance of AHU, Moreover, we propose a NN controller for the outer control loop to enhance the adaptive ability and robustness of the entire control loop. The NN is trained online with the SPSA-based training algorithm with the guarantee of convergence and stability. Realtime experiment results demonstrate the performance of the new cascade control scheme. The temperature control performance of AHU is significantly improved compared with the classical PID control system both for temperature set-point tracking and chilled water flow rate disturbances rejection.

\section{REFERENCES}

[1] J. E. Seem, "A new pattern recognition adaptive controller with application to HVAC systems," Automatica, vol. 34, no. 8, pp. 969-982, 1998.
[2] G. Stephanopoulos, Chemical Process Control: An Introduction to the Theory and Practice. Englewood Cliffs, NJ: Prentice-Hall, 1984.

[3] P. W. Murrill, Application Concepts Process Control. RTP, North Carolina: ISA, 1988.

[4] C. Maffezzoni, N. Schiavoni, and G. Ferretti, "Robust design of cascade control," IEEE Control Syst. Mag., pp. 21-25, Jan. 1990.

[5] R. C. Miller and J. E. Seem, "Comparison of artificial neural networks with traditional methods of predicting return time from night or weekend setback," Trans. ASHRAE, vol. 97, no. 2, 1991.

[6] P. S. Curtiss, J. F. Kreider, and M. J. Brandemuehl, "Adaptive control of HVAC processes using predictive neural networks," Trans. ASHRAE, vol. 100, no. 1, pp. 36-46, 1994.

[7] S. J. Hepworth and A. L. Dexter, "Neural control of nonlinear HVAC plant," in Proc. IEEE Conf. Control Appl., 1994, vol. 3, pp. 368-376.

[8] M. Heiss, "Error-minimizing dead zone for basis function networks," IEEE Trans. Neural Netw., vol. 7, no. 6, pp. 39-50, Nov. 1996.

[9] Q. Song, J. C. Spall, and Y. C. Soh, "Robust neural network tracking controller using simultaneous perturbation stochastic approximation," in Proc. 42nd IEEE Conf. Decision and Control, Maui, HI, Dec. 2003, pp. 6194-6199.

[10] J. C. Spall, "Multivariate stochastic approximation using a simultaneous perturbation gradient approximation," IEEE Trans. Autom. Control, vol. 37, no. 3, pp. 332-341, Mar. 1992.

[11] J. C. Spall and J. A. Cristion, "Model free control of nonlinear stochastic systems with discrete-time measurements," IEEE Trans. Autom. Control, vol. 43, no. 9, pp. 1198-1210, Sep. 1998.

[12] J. C. Spall, "An overview of the simultaneous perturbation method for efficient optimization," in Johns Hopkins APL Technical Digest, 1998, vol. 19, pp. 482-492.

[13] G. H. Cohen and G. A. Coon, "Theoretical consideration of retarded control," Trans. ASME, vol. 75, pp. 827-834, 1953.

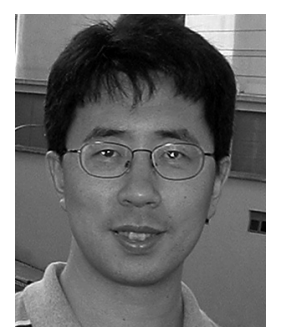

Chengyi Guo received the B.E. degree from the University of Electronic Science and Technology of China, Chengdu, in 1997. He is currently working towards the Ph.D. degree in the School of Electrical and Electronic Engineering, Nanyang Technological University, Singapore.

His research interests include robust control, neural network controllers, and optimization theory. 


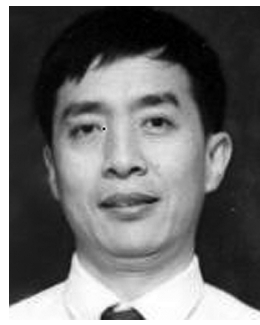

Qing Song (S'88-M'91) received the B.S. degree from the Harbin Shipbuilding Engineering Institute, Heilongjiang, China, in 1982, the M.S. degree from the Dalian Maritime University, Dalian, China, in 1986, and the Ph.D. degree from the Industrial Control Centre, Strathclyde University, Glasgow, U.K., in 1992.

He was an Assistant Professor at the Dalian Maritime University from 1986 to 1987 . He worked as a Research Engineer at the System and Control Pte Ltd., U.K., before he joined the School of Electrical and Electronic Engineering, Nanyang Technological University, as a Lecturer in 1992. He is now an Associate Professor. He is also an active industrial consultant. He is the first inventor of a neural network related patent and was the recipient of several Academic Exchange Fellowships with more than 30 referred international journal publications. He has been the principal investigator of a few research programs targeted to industry in the area of computational intelligence.

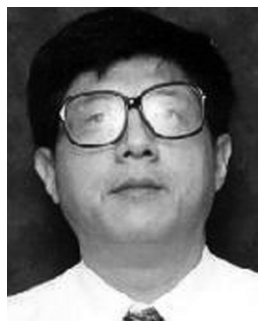

Wenjian Cai (M'05) received the B.Eng. and M.Eng. degrees from the Department of Precision Instrumentation Engineering and the Department of Control Engineering, Harbin Institute of Technology, Heilongjiang, China, in 1980 and 1983, respectively, and the Ph.D. degree from the Department of Electrical Engineering, Oakland University, Rochester, MI, in 1992.

After graduation, he worked as a Postdoctoral Research Fellow at the Center for Advanced Robotics, Oakland University, Research Scientist at the National University of Singapore, Principal Engineer and R\&D Manager at Supersymmetry Services Pte Ltd., and Senior Research Fellow at the Environmental Technology Institute. He is currently an Associate Professor at the Nanyang Technological University, Singapore. He has more than 20 years of industry and research experience in the areas of environmental and energy conservation technologies. He participated in many industry related research projects and published more that 80 technical papers. His current research interests are sensoring and control technologies in environmental and energy processes, multivariable control, and identification. 